\title{
Constraining simulated black hole feedback in the Perseus Cluster
}

\author{
Andrew M. Heinrich ${ }^{1,2}$, Yi-Hao Chen ${ }^{1}$, Sebastian Heinz ${ }^{1}$, Irina Zhuravleva ${ }^{2}$, and Eugene Churazov $^{3}$ \\ ${ }^{1}$ Department of Astronomy, University of Wisconsin-Madison, Madison, WI, USA \\ ${ }^{2}$ Department of Astronomy and Astrophysics, University of Chicago, Chicago, IL, USA \\ ${ }^{3}$ Max Planck Institute for Astrophysics, Garching, Bavaria, Germany
}

\begin{abstract}
Active galactic nuclei in galaxy clusters can produce tightly collimated jets that inject energy into the surrounding intracluster medium (ICM). This feedback process can offset cooling losses from X-ray radiation that otherwise would result in cooling flow structures. Jet-inflated bubbles can heat the cluster via a turbulent cascade. The density fluctuation spectrum inferred from X-ray maps of the cluster shows different levels and scales of turbulence in different regions of the clusters, and can be used to determine the contribution of different driving mechanisms. By applying observational power spectrum techniques to synthetic X-ray data from feedback simulations of the Perseus cluster, we find that the density fluctuation spectrum produced by a single episode of jet activity are broadly consistent with analysis of Chandra observations inside $60 \mathrm{kpc}$. The velocity dispersion effected by the AGN approaches Hitomi's measurement of turbulence in Perseus during the AGN episode, but is underpredicted later in the simulation. A fully established volume-filling turbulent cascade therefore requres multiple periods of jet activity, along with turbulence driven by other processes outside of the inner $60 \mathrm{kpc}$ core. This also suggests the X-ray fluctuations directly associated with the jet bubble substantially contribute to the overall density fluctuation spectrum. ${ }^{1}$
\end{abstract}

\section{Introduction}

Galaxy clusters are among the most massive objects yet observed by extragalactic astronomers. Among these objects none is better studied than Abell 426, located in the Perseus constellation. Perseus is a rich cluster, with more than a thousand individual galaxies gravitationally bound together. The galaxies orbit through a vast atmosphere of hot, diffuse gas known as the intracluster medium, hereafter ICM. The ICM typically reaches temperatures between $10^{7}$ and $10^{8} \mathrm{~K}$ (Fabian and Nulsen, 1977) and dominates the baryonic mass distribution of clusters (Chiu et al., 2015). This high-temperature plasma shines brightly in the X-ray band (Figure 1), with cooling timescales significantly shorter than cluster ages (Fabian, 1994). Thus cool-core clusters like Perseus should form a cooling flow structure wherein an order of 100 solar masses per year should coalesce and contribute to star formation. Observations of Perseus and other large clusters like it show a large discrepancy between this prediction and observed rates of star formation. X-ray spectroscopic analysis also shows a distinct lack of low-temperature ions that one would expect to be present (Peterson et al., 2002). This cooling flow problem has been central to the study of galaxy clusters for decades.

This 'cooling flow gap' must be due to an external heat source acting on the cluster. Several candidate sources exist that can each contribute to this discrepancy. Cosmic ray streaming (Ehlert et al., 2018), thermal conduction (Yang and Reynolds, 2016), and sloshing caused by subcluster mergers (Motl et al., 2004) have all been suggested and studied in detail, each acting most strongly on different regions of the cluster. The most promising source of energy to explain the inner core of clusters is active galactic nucleus (AGN) feedback (Churazov et al., 2000). The brightest central galaxy, NGC 1275 in the case of the Perseus cluster, contains a supermassive black hole (SMBH) that launches a jet into the ICM. The jet material likely comes from the accretion disc of the AGN, and while the exact mechanism that produces the jet is yet to be fully understood, it is likely tied to strong magnetic fields around the SMBH. This jet inflates a bubble that shines brightly in the radio band due to synchrotron emission, and can be seen as cavities in X-ray maps of the ICM. Looking at Perseus (Figure 1), multiple sets of these bubbles can be seen owing to multiple episodes of jet activity. The structures closest to the core of the cluster are dominated by the jet, while the outer structures of the cluster can mostly be attributed to sloshing from an ongoing galaxy merger (Walker et al., 2018).

\footnotetext{
${ }^{1}$ This work documents a presentation following Heinrich et al. (2021) given at the 31st Annual Wisconsin Space Conference.
} 


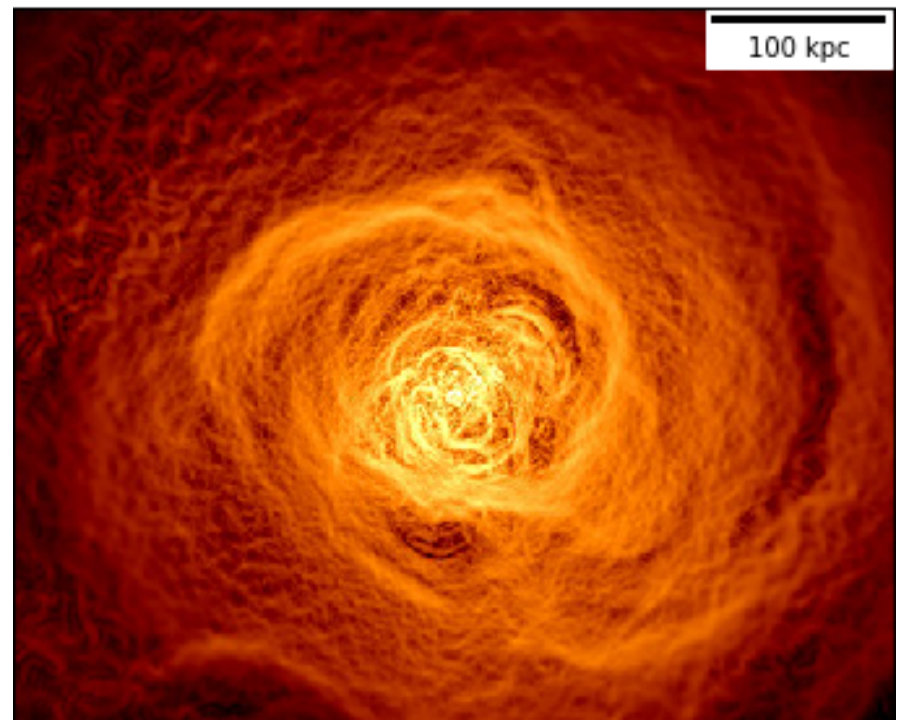

Figure 1: The Perseus Cluster (Abell 426), the brightest cluster in the X-ray sky. Shown here is an X-ray image of Perseus composed of data from the Chandra X-ray Observatory treated with a Gaussian Gradient Magnitude (GGM) filter, which shows edges in the surface brightness (Sanders et al., 2016).

Measurements of turbulent heating in Perseus have been taken from several datasets. Notably, Perseus was the target of the Hitomi observatory's only successful observation (Hitomi Collaboration, 2016). One of the significant results from this observation was a measurement of the small-scale velocity dispersion in Perseus of $164 \pm 10 \mathrm{~km} / \mathrm{s}$, taken from ionized iron lines in the produced X-ray spectrum. After this observation, the satellite broke up in orbit.

Even prior to this measurement, there was a push among galaxy cluster astrophysicists to calculate the turbulent velocities in clusters. The seminal method used to quantify turbulence in X-ray images of the ICM without high quality spectroscopic data was to calculate amplitudes of X-ray surface brightness fluctuations via a power spectrum. After removing large scale gradients from X-ray maps taken from Chandra, Zhuravleva et al. (2015) calculated the density fluctuation spectrum of multiple regions of the cluster, starting from the core and moving outwards. They found X-ray surface brightness fluctuations in broad agreement with Hitomi's measurement, which is sufficient to balance radiative cooling, therefore potentially explaining the cooling flow gap (Zhuravleva et al., 2014).

As there are several processes acting on Perseus, it is difficult to separate the contributions of each candidate process to the observed cluster heating. We thus turn to high resolution three-dimensional simulations to isolate sources of turbulence. Using this same power spectrum method, we can decompose the observed density fluctuations into their component sources. It has been shown via this method that merger driven sloshing can entirely explain brightness fluctuations outside of the inner $60 \mathrm{kpc}$ core of Perseus (ZuHone et al., 2018; Walker et al., 2018). It is thus theorized that AGN jets in the Perseus cluster must account for the bulk of X-ray fluctuations inside the $60 \mathrm{kpc}$ limit. We use a simulated jet modelled off the Perseus Cluster to constrain the source of turbulent heating. We calculate the power spectrum of synthetic X-ray maps via the $\Delta$-variance method (Arévalo et al., 2012).

\section{Methods}

We use the FLASH code (Dubey et al., 2009) to model and AGN jet firing for $10 \mathrm{Myr}$ at the center of a spherically symmetric atmosphere. The cluster atmosphere is set with density and temperature tuned to match the parameters found for the Perseus cluster according to the widely used $\beta$-model that describes relaxed clusters (Chen et al., 2019). The jet itself is modelled as a nozzle through which mass, momentum, energy, and magnetic flux are fired into the atmosphere in both the positive and negative z-directions. These simulations do not model the formation of the jet itself, rather they assume that the jet already exists and simulate the interaction between the jet and its environment. The nozzle wobbles slightly arround its axis to account for the "dentist drill" effect (Scheuer, 1982). 
These simulations employ an adaptive mesh refinement (AMR) grid structure, wherein the resolution of the cluster gas changes in different regions and times throughout the simulation domain. The refinement of the simulation is increased in areas of high activity and relaxed in areas of low activity in order to maintain computational efficiency. In order to properly resolve the tightly collimated jets, the smallest resolution element while the jet is firing is 30 $\mathrm{pc}$, which increases to $120 \mathrm{pc}$ after the jet activity ends. Our numerical resolution significantly exceeds the imaging resolution of Chandra's observations of Perseus.

Using the yt python package (Turk et al., 2010), we produce synthetic X-ray images of the simulated cluster by projecting the X-ray emissivity along the coordinate axes. The emissivity is calculated between 0.5 and $3.5 \mathrm{keV}$ (the energy band used by Chandra) assuming the cluster contains half the solar metallicity (Smith et al., 2001). These images are then normalized following the method applied to the Chandra data. We fit the image to the azimuthally averaged $\beta$-model, which removes large scale fluctuations that would otherwise dominate the power spectrum. Figure 2 shows several timesteps of the jet simulation after the X-ray surface brightness maps have been normalized. The fit is accomplished via a Monte Carlo markov chain (MCMC) sampler, finding parameters for the surface brightness to match the beta model (Equation 1), including the central brightness $S_{0}$, core radius $r_{c}$, and the $\beta$ parameter.

$$
S=S_{0}\left[1+\left(\frac{r}{r_{c}}\right)^{2}\right]^{-3 \beta+0.5}
$$

As the AMR grid changes over the course of the simulation, the normalized X-ray surface brightness images can contain grid artifacts at large radii, corresponding to large resolution elements at the outer reaches of the simulation domain. These artifacts can be removed via a smoothed covering grid, which interpolates across cell boundaries and allows us to produce X-ray maps with fewer numerical artifacts.

To quantitatively measure the surface brightness fluctuations, we divide the cluster into equally spaced annuli with widths of $30 \mathrm{kpc}$. On the sky, these would correspond to 1.5 arcminute wide rings centered on the cluster core. We then employ the modified $\Delta$-variance method (Arévalo et al., 2012) to find the power spectrum inside each annulus (see also Stutzki et al. (1998) for the original version of this method). The $\Delta$-variance method is used over the more standard fast Fourier transform in this implementation because masking the image in order to separate each annuli would otherwise create large edge artifacts in the resultant power spectrum. This method convolves the normalized images with a modified Mexican Hat filter as in Equation 2, with the image I, mask M, and two closely separated Gaussian filters corresponding to a spatial scale $\sigma$.

$$
F=\left(\frac{G_{\sigma 1} * I}{G_{\sigma 1} * M}-\frac{G_{\sigma 2} * I}{G_{\sigma 2} * M}\right) M
$$

We then integrate over the square of the result to yield the Variance $V_{k}$, which can be used to calculate the power spectrum $P(k)$ over any scale factor (Equation 3 ).

$$
V_{k}=\int(F * I)^{2} d^{2} x=P(k) \epsilon^{2} \pi k^{2}
$$

This variance method essentially allows us to select for different scales of detail in an image. This is demonstrated in Figure 3. The resultant power spectrum is then converted to the three-dimensional density fluctuation spectrum (Zhuravleva et al., 2015). We calculate each spectrum for wavefactors corresponding to scales between 3 and $150 \mathrm{kpc}$ in Perseus.

As has been previously stated, the Perseus cluster contains at least two sets of cavities corresponding to multiple episodes of past jet activity. Any single simulation time, each containing one jet, will therefore not be able to account for the surface brightness fluctuations observed by Chandra. We attempt to approximate a complex jet formation history by combining power spectra from different simulation times. Using a parameter space of $10 \mathrm{Myr}$ increments from 10 to $80 \mathrm{Myr}$, we use an MCMC algorithm (Foreman-Mackey et al., 2012) to find the best-fit linear combination of our synthetic spectra to observed density fluctuations in Perseus. Because the atmosphere outside of $60 \mathrm{kpc}$ can be turbulently heated by merger-driven sloshing (Walker et al., 2018), we focus our fit on the inner two annuli, between 0 and $60 \mathrm{kpc}$, and ensure that the simulations don't overpredict the outer annuli. 


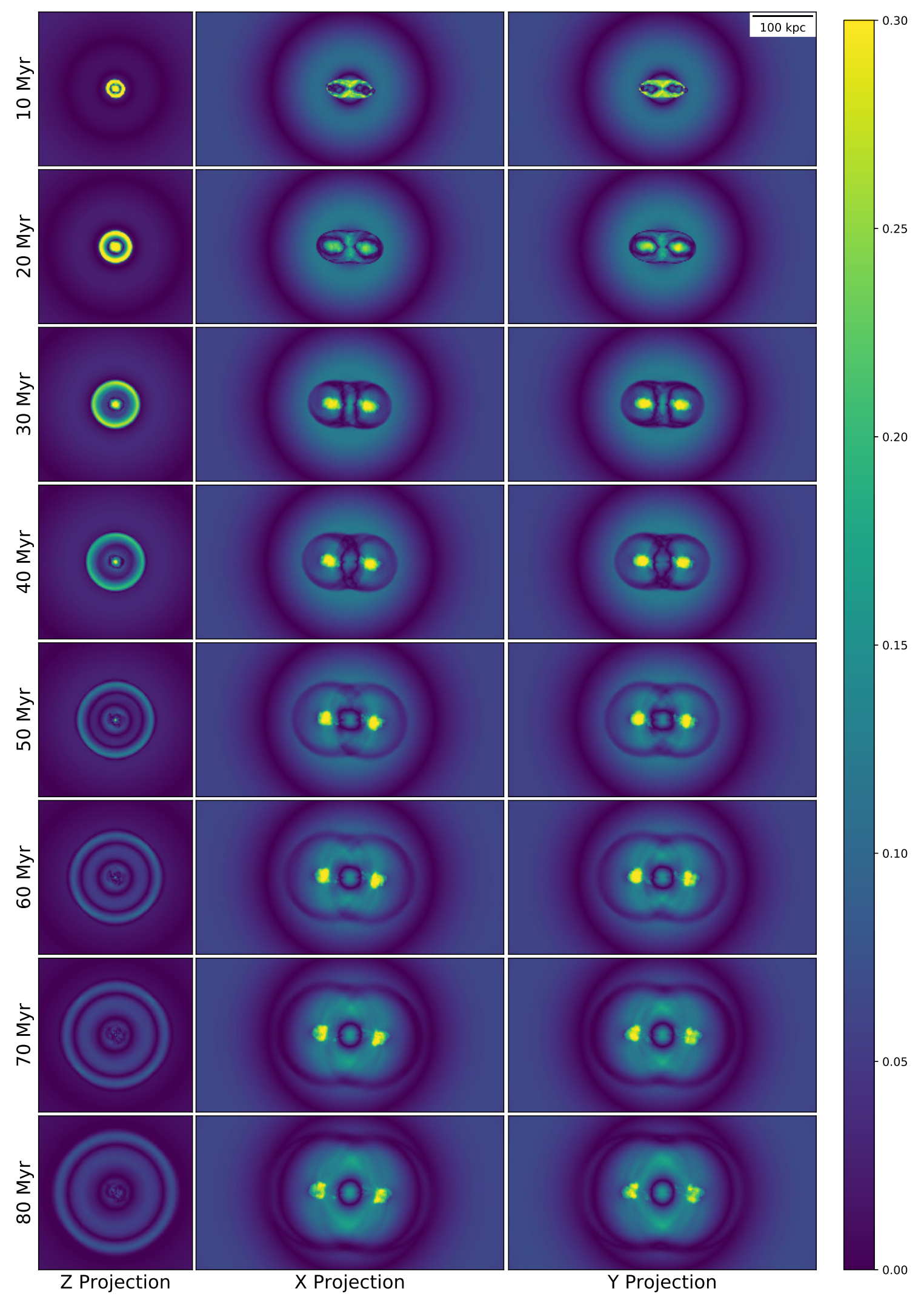

Figure 2: Normalized X-ray surface brightness maps of the simulation after the removal of the $\beta$-model. Eight different simulation times, between 10 and $80 \mathrm{Myr}$ after the jet begins firing, are shown at three viewing angles ( $\mathrm{x}-, \mathrm{y}$ - and $\mathrm{z}$-directions). The $\beta$-model is fitted to each timestep individually (Heinrich et al., 2021). 


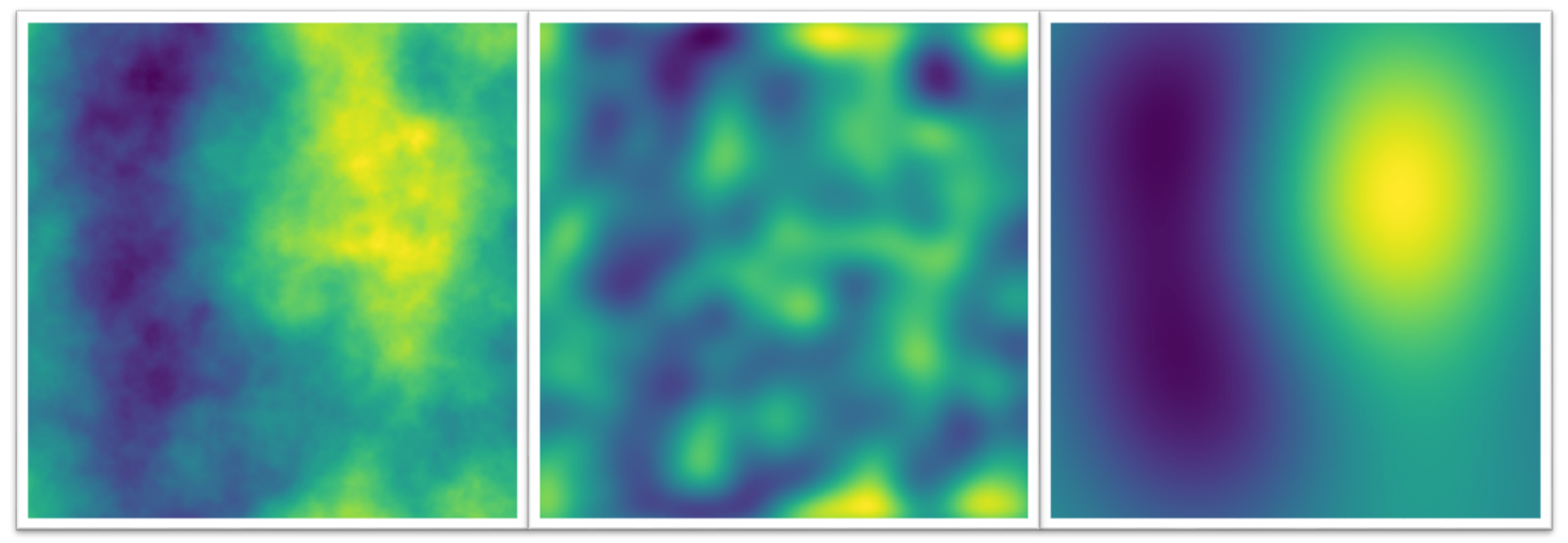

Figure 3: A demonstration of the $\Delta$-variance method. Left: A randomly generated image with an injected power-law spectrum $\left(P(k) \propto k^{-3}\right)$. Center: The variance of the generated image at a small spatial scale (large $\left.\mathrm{k}\right)$. Right: The variance of the generated image at a large spatial scale (small k). The variance method selects for different sizes of structure given a specific value of $\mathrm{k}$.

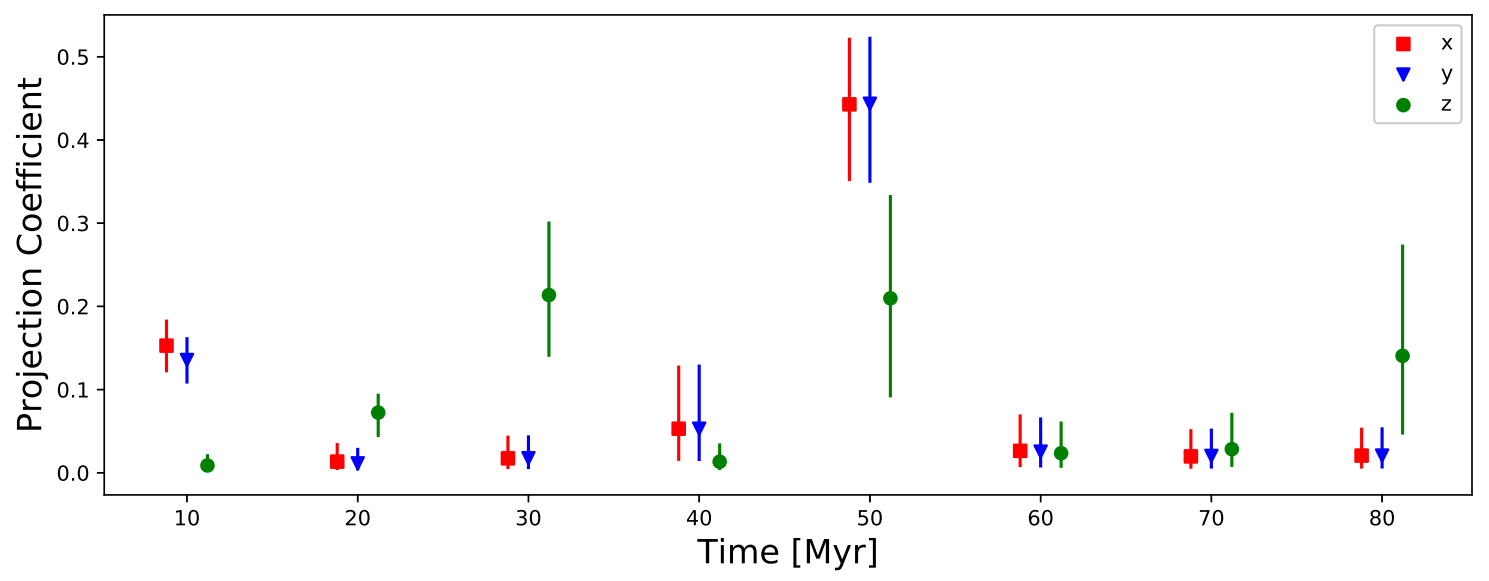

Figure 4: Weighting coefficients for each sampled power spectrum in three viewing angles.

\section{Results and Discussion}

The density flucuation spectra produced from our simulations show a wide variety of power throughout the sampled scale factors, annuli, and simulation times. The progression of the jet impact through the cluster can clearly be seen propagating throughout the annuli. At early times the power spectral signature of the jet can reach up to three times the power observed by Chandra, but this quickly fades. In the outer annuli, the simulated spectra only very briefly exceed Chandra observations, and only do so if the observer is stationed along the axis of the jet.

Using our MCMC fitting algorithm to attempt to match the inner $60 \mathrm{kpc}$ core's fluctuations, we find the best-fit weighting coefficients for each sampled power spectrum. In the off-axis ( $\mathrm{x}$ and $\mathrm{y}$ ) projections, which are very selfsimilar, the best fit comes from a combination of jets aged 10 and 50 Myr. The on-axis projection uses the 30,50 and $80 \mathrm{Myr}$ projection (Figure 4).

The resultant fitted power spectra are shown in Figure 5. The MCMC algorithm provides a much better match to observational data than is possible with a single snapshot of the simulation. There are still major discrepancies between the simulation and observed behavior of the cluster atmosphere. The spectra are typically suppressed at low values of $\mathrm{k}$, and peak at slightly smaller spatial scales. This may be due to the lack of a large scale asymmetry in the simulations 


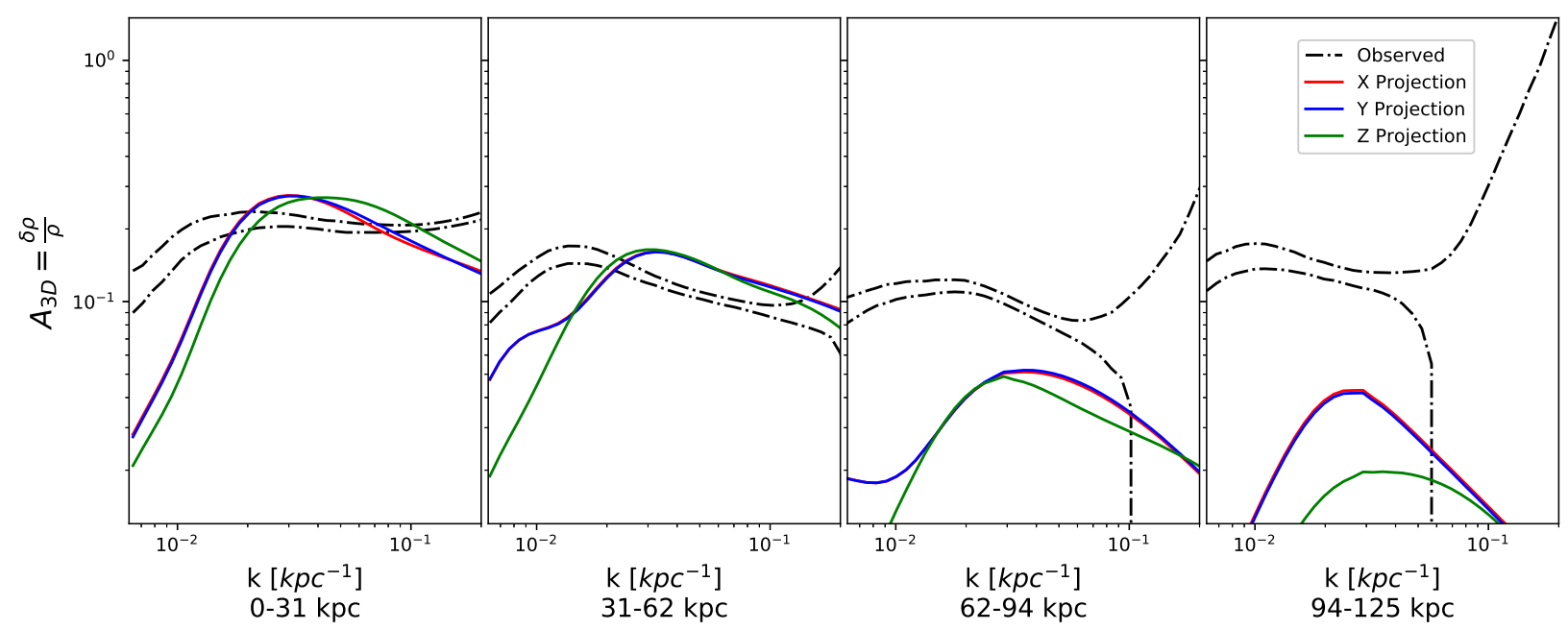

Figure 5: Combined weighted power spectrum at three viewing angles, overplotted on observed minimum and maximum power spectral density (PSD) (Zhuravleva et al., 2015).

which is present in Chandra observations.

Additionally, given a measured sound speed in Perseus we can use our density fluctuation spectra to estimate the turbulence in Perseus. We find that our spectra (when converted to velocity units) are on the same order of magnitude of the Hitomi observation of turbulence in the cluster. However, when the RMS velocity in the simulations are directly measured, early times of the simulation approach the Hitomi measurement and later times consistently underpredict this value. This suggests that the X-ray surface brightness fluctuations contain contributions from established turbulence as well as other structures (shocks and bubbles). It has been found that excising the sharp edges created by X-ray cavities can reduce the PSD of intensity fluctuations by up to a factor of 2 in Chandra observations (Zhuravleva et al., 2015).

Our simulations support the suggestion that the surface brightness fluctuations in the inner $60 \mathrm{kpc}$ region of Perseus must rely on AGN feedback, though our diagnostic cannot uniquely constrain the injection history of jet power. Our method of linearly combining jet ages depends on an assumption that the simulation's perturbations are sufficiently small such that the power spectra can be combined in this way. Notably, this assumption ignores interaction between individual jet bubbles, but should still produce viable results if AGN duty cycles are on the order of $10 \%$. Simulations that contain multiple episodes of jet activity natively would remove this uncertainty, however these are costly to run at this resolution and require knowledge of jet ages in Perseus. It is also important to note that jet inclination in Perseus is not well constrained. This work views the jet both from an on-axis observer as well as two perpendicular ones, but in reality the inclination will lie somewhere in between.

\section{Conclusions}

By performing equivalent analysis on observed and synthetic images of the Perseus cluster, we calculate the $\Delta$ variance power of X-ray emission from the ICM. Simulating a single episode of an AGN jet allows us to isolate its contribution to the cluster atmopshere as a whole.

- X-ray derived density fluctuations in the ICM are comparable in magnitude and spectral form within $60 \mathrm{kpc}$ from the cluster center.

- PSD values can trace the propagation of jet-inflated bubbles and decline after its passing, leaving behind a turbulent ICM.

- A linear combination of power spectra can improve the reproduction with two or three episodes of jet activity. 
- Our results complement similar analysis of sloshing simulations, wherein it is determined that sloshing dominates the PSD outside of $60 \mathrm{kpc}$. The AGN is responsible for intensity fluctuations within this limit.

- The line-of-sight projected single component velocity dispersion is significantly underpredicted by our numerical model, indicating a single AGN episode (as modelled in our work) does not produce the volume-filling level of turbulence measured by Hitomi.

\section{Acknowledgments}

AH would like to thank the Wisconsin Space Grant Consortium for their support via NASA Training Grant NNX15AJ12H. Data used in this work was originally produced under the Astrophysics Theory Program grant NNX17AJ98G and the National Science Foundation through grant AST1616101. This work used the Extreme Science and Engineering Discovery Environment (XSEDE) Stampede at the Texas Advanced Computing Center at The University of Texas at Austin and the HPC Cluster at the Center for High Throughput Computing at the University of Wisconsin-Madison. Support for this research was also provided by the Hilldale Undergraduate Research Fellowship.

\section{References}

Arévalo, P., Churazov, E., Zhuravleva, I., Hernández-Monteagudo, C., and Revnivtsev, M. (2012). A Mexican hat with holes: calculating low-resolution power spectra from data with gaps. Monthly Notices of the Royal Astronomical Society, 426(3):1793-1807.

Chen, Y. H., Heinz, S., and Enßlin, T. A. (2019). Jets, bubbles, and heat pumps in galaxy clusters. Monthly Notices of the Royal Astronomical Society, 489(2):1939-1949.

Chiu, I., Mohr, J., McDonald, M., Bocquet, S., Ashby, M. L. N., Bayliss, M., Benson, B. A., Bleem, L. E., Brodwin, M., Desai, S., Dietrich, J. P., Forman, W. R., Gangkofner, C., Gonzalez, A. H., Hennig, C., Liu, J., Reichardt, C. L., Saro, A., Stalder, B., Stanford, S. A., Song, J., Schrabback, T., Šuhada, R., Strazzullo, V., and Zenteno, A. (2015). Baryon content of massive galaxy clusters at $0.57 \leq \mathrm{z} \leq 1.33$. Monthly Notices of the Royal Astronomical Society, 455(1):258-275.

Churazov, E., Forman, W., Jones, C., and Böhringer, H. (2000). Asymmetric, arc minute scale structures around NGC 1275. Astronomy and Astrophysics, 356:788-794.

Dubey, A., Reid, L. B., Weide, K., Antypas, K., Ganapathy, M. K., Riley, K., Sheeler, D., and Siegal, A. (2009). Extensible Component Based Architecture for FLASH, A Massively Parallel, Multiphysics Simulation Code. Parallel Computing, 35(10-11):512-522.

Ehlert, K., Weinberger, R., Pfrommer, C., Pakmor, R., and Springel, V. (2018). Simulations of the dynamics of magnetized jets and cosmic rays in galaxy clusters. Monthly Notices of the Royal Astronomical Society, 481(3):2878-2900.

Fabian, A. C. (1994). Cooling Flows in Clusters of Galaxies. Annual Review of Astronomy and Astrophysics, 32:277318.

Fabian, A. C. and Nulsen, P. E. J. (1977). Subsonic accretion of cooling gas in clusters of galaxies. Monthly Notices of the Royal Astronomical Society, 180(3):479-484.

Foreman-Mackey, D., Hogg, D. W., Lang, D., and Goodman, J. (2012). emcee: The MCMC Hammer. Publications of the Astronomical Society of the Pacific, 125(925):306-312.

Heinrich, A. M., Chen, Y.-H., Heinz, S., Zhuravleva, I., and Churazov, E. (2021). Constraining black hole feedback in galaxy clusters from X-ray power spectra. Monthly Notices of the Royal Astronomical Society, 505(3):4646-4654.

Hitomi Collaboration (2016). The quiescent intracluster medium in the core of the Perseus cluster. Nature, 535(7610):117-121.

Motl, P. M., Burns, J. O., Loken, C., Norman, M. L., and Bryan, G. (2004). Formation of Cool Cores in Galaxy Clusters via Hierarchical Mergers. The Astrophysical Journal, 606(2):635-653. 
Peterson, J. R., Kahn, S. M., Paerels, F. B. S., Kaastra, J. S., Tamura, T., Bleeker, J. A. M., Ferrigno, C., and Jernigan, J. G. (2002). High Resolution X-ray Spectroscopic Constraints on Cooling-Flow Models for Clusters of Galaxies. The Astrophysical Journal, 590(1):207-224.

Sanders, J. S., Fabian, A. C., Russell, H. R., Walker, S. A., and Blundell, K. M. (2016). Detecting edges in the X-ray surface brightness of galaxy clusters. Monthly Notices of the Royal Astronomical Society, 460(2):1898-1911.

Scheuer, P. A. G. (1982). Morphology and power of radio sources. Extragalactic radio sources; IAU Symposium, 97:163-165.

Smith, R. K., Brickhouse, N. S., Liedahl, D. A., and Raymond, J. C. (2001). Collisional Plasma Models with APEC/APED: Emission-Line Diagnostics of Hydrogen-like and Helium-like Ions. The Astrophysical Journal, 556(2):L91-L95.

Stutzki, J., Bensch, F., Heithausen, A., Ossenkopf, V., and Zielinsky, M. (1998). On the fractal structure of molecular clouds. Astronomy and Astrophysics, 336:697-720.

Turk, M. J., Smith, B. D., Oishi, J. S., Skory, S., Skillman, S. W., Abel, T., and Norman, M. L. (2010). yt: A MULTI-CODE ANALYSIS TOOLKIT FOR ASTROPHYSICAL SIMULATION DATA. The Astrophysical Journal Supplement Series, 192(16pp):9.

Walker, S. A., Sanders, J. S., and Fabian, A. C. (2018). What fraction of the density fluctuations in the Perseus cluster core is due to gas sloshing rather than AGN feedback? Monthly Notices of the Royal Astronomical Society, 481(2):1718-1725.

Yang, H. Y. K. and Reynolds, C. S. (2016). How AGN Jets Heat the Intracluster Medium-Insights from Hydrodynamic Simulations. The Astrophysical Journal, 829(2):90.

Zhuravleva, I., Churazov, E., Arévalo, P., Schekochihin, A. A., Allen, S. W., Fabian, A. C., Forman, W. R., Sanders, J. S., Simionescu, A., Sunyaev, R., Vikhlinin, A., and Werner, N. (2015). Gas density fluctuations in the Perseus Cluster: clumping factor and velocity power spectrum. Monthly Notices of the Royal Astronomical Society, 450(4):4184-4197.

Zhuravleva, I., Churazov, E., Schekochihin, A. A., Allen, S. W., Arévalo, P., Fabian, A. C., Forman, W. R., Sanders, J. S., Simionescu, A., Sunyaev, R., Vikhlinin, A., and Werner, N. (2014). Turbulent heating in galaxy clusters brightest in X-rays. Nature, 515(7525):85-87.

ZuHone, J. A., Kowalik, K., Öhman, E., Lau, E., and Nagai, D. (2018). The Galaxy Cluster Merger Catalog: An Online Repository of Mock Observations from Simulated Galaxy Cluster Mergers. The Astrophysical Journal Supplement Series, 234(1):4. 